\title{
A Validated Study of a Modified Shallow Water Model for Strong Cyclonic Motions and Their Structures in a Rotating Tank
}

\author{
Hung-Cheng Chen $\mathbb{D}^{1},{ }^{1}$ Jai-Houng Leu $\mathbb{D}^{1},{ }^{1}$ Yong Liu $\left(\mathbb{D},{ }^{1}\right.$ He-Sheng Xie ${ }^{\mathbb{D}}{ }^{1}$ \\ and Qiang Chen $\mathbb{D i D}^{2}$
}

${ }^{1}$ School of Intelligent Manufacturing, Shandong Polytechnic, Jinan 250104, China
${ }^{2}$ School of Railway, Shandong Polytechnic, Jinan 250104, China

Correspondence should be addressed to Jai-Houng Leu; jahonleu@yahoo.com.tw

Received 30 January 2021; Revised 29 March 2021; Accepted 20 April 2021; Published 3 May 2021

Academic Editor: Teen-Hang Meen

Copyright ( $\odot 2021$ Hung-Cheng Chen et al. This is an open access article distributed under the Creative Commons Attribution License, which permits unrestricted use, distribution, and reproduction in any medium, provided the original work is properly cited.

\begin{abstract}
A joint theoretical and numerical study was carried out to investigate the fluid dynamical aspect of the motion of a vortex generated in a rotating tank with a sloping bottom. This study aims at understanding the evolution of strong cyclonic motions on a $\beta$-plane in the Northern Hemisphere. The strong cyclonic vortices were characterized by four nondimensional parameters which were derived through a scale analysis of the depth variations of fluid. By simplifying the model flow field and the prototype flow field, respectively, through the conservation of potential vorticity, two sets of dynamic similarity conditions are derived. This study proposed a sophisticated modified shallow water model (MSWM) to investigate the flow features of such strong vortices. A detailed numerical calculation adopted by multidimensional positive definite advection transport algorithm (MPDATA) was carried out to validate those effects considered in the MSWM model, including sloping bottom, parabolic free surface deformation, and viscous dissipation. Close agreements were found between the experimental and numerical results, including the streamlines patterns and the vortex trajectory. Comprehensive simulations for strong cyclonic vortices over different sloping bottoms were investigated to understand the impact of planetary $\beta$ effect on vortex. The results calculated by MSWM demonstrate a variety of flow features of interactions between the primary vortex and induced secondary Rossby wave wakes that were essential and prominent in environmental geophysical flows.
\end{abstract}

\section{Introduction}

A joint theoretical and numerical study is used to investigate the fluid dynamical aspect of the motion of a vortex generated in a rotating tank with a sloping bottom. This study is motivated by getting an insight of the evolution of a strong barotropic cyclone on a $\beta$-plane in the Northern Hemisphere. The dynamics of barotropic vortices on a $\beta$-plane have been studied intensively over the past several decades through analytical, numerical, and laboratory investigations [1-22]. Both analytical and numerical studies have shown that (a) a $\beta$-gyre develops in the initial time [1-4], (b) the vortex intensity or structure changed during the vortex propagation [5-9], and (c) a more intense quasi-geostrophic vortex may evolve for a longer time [10]. These dynamic features are basically examined by the advancement of the fluid cyclonically around the vortex core. It is considered to be a nonlinear, self-propelling motion or the interaction between $\beta$-gyre and vortex with time evolution under the effect of Rossby wave radiation.

The above phenomena can be further elucidated from the viewpoint of point vortex dynamics. In many studies, Resnik et al. [10] presented the theory of long-term evolution of singular or point vortex based on the conservation of vortex energy and enstrophy, which is of great significance. They suggest that the evolution of intense vortices on the $\beta$-plane can be divided into three stages. In the first stage, the beta-gyres generated by the near-field radiation of Rossby wave and the nonlinear advection of the vortex make the cyclone (anticyclone) move to the northwest (southwest). The study of vortex dynamics at this stage has attracted much attention, including laboratory experiments [11-13], 
numerical simulation $[3,4,18,19]$, and theoretical analysis $[5-7,9]$. In the second stage, the amplitude and velocity of the vortex are gradually slowed down by other azimuthal harmonics generated by Rossby wave radiation and nonlinearity. In the last third stage, the amplitude of the vortex decreases to the background value under the continuous influence of Rossby wave radiation. The above three stages correspond to three characteristic scales of time. They are advection time scale, wave time scale, and distortion time scale. For a detailed review of the vortex dynamics of singular vortices on the $\beta$-plane, the reader is referred to the work of Resnik \& Kravtsov [20].

In the laboratory experiments, Firing and Beardsley [11] conducted the first laboratory experiment of a barotropic eddy on a $\beta$-plane. By generating the eddy with a piston mechanism on an inclined plate with a gentle slope $\left(s_{y} \approx 0.1\right)$, these authors observed that the eddy evolved to form a dipole in a short time. Their purpose was to validate the initial northwestward translation of the vortex on a $\beta$-plane which was predicted earlier by Adem [1]. To generate a longlived isolated eddy, Takematsu and Kita [12] applied a locally cooling method to create a monopolar vortex on an inclined bottom in a rotating fluid. The resulting stratified eddy was inherently stable and migrated to the northwest as a recognizable structure like a Gulf Stream ring. Masuda et al. [13] carried out a joint laboratory and numerical study for a strong isolated eddy on an inclined plate with a steep slope $\left(s_{y} \approx 0.33\right)$. Satisfactory agreements were obtained on the flow patterns between the laboratory and numerical experiments. They adopted a two-dimensional quasigeostrophic vorticity equation (QGVE) to numerically simulate the flow specifically for a small Rossby number vortex.

The initial vortex conditions for QGVE simulation were carefully estimated by measuring water column shrinking or stretching in a sink/source vortex generator. The results showed that the vortex evolved into a larger main eddy, and several secondary eddies displayed significant influence of the strong $\beta$ effect on the cyclonic vortex.

For a long-lived vortex translating on a gentle-slope $\left(s_{y} \approx 0.13\right)$ bottom, Carnevale et al. [14] generated isolated vortices using stirred/sink methods and numerically solved a QGVE to simulate their motions. They interpolated both by a Gaussian-type distribution and a Rankine-type distribution as the initial conditions of numerical integrations. The results showed that the essential mechanism of both the stirring-induced and the sink-induced vortices is inviscid, quasi-geostrophic. Flór and Eames [15] investigated the dynamics of a cyclonic monopolar vortex on a topographic $\beta$-plane by laboratory experiments and theoretical analysis. They systematically measured the vortex distributions generated by the stirred or the suction method and characterized the initial distribution in terms of a radius $R_{m}$, the maximum azimuthal velocity $v_{\theta}$, and a dimensionless parameter $\alpha$ which describes the steepness of the velocity profile.

Recently, Chen et al. [22] generated a strong barotropic vortex of large vortex Rossby number $\mathrm{Ro}_{v} \sim O(1)$ in a rotating tank with a gentle sloping bottom $\left(s_{y} \approx 0.0538\right)$ to simulate the movement of a hurricane-like vortex on a $\beta$-plane. The cyclonic vortex was generated by a rotating cylinder in a thin-walled hollow cylinder in parallel to the axis of the rotating tank. The most remarkable feature of this study is the use of gradient wind balance vortex model to capture vortex structure. The radial distribution of depression depth on the vortex surface was clearly visualized by the illumination of a laser light sheet perpendicular to the vortex centre. The parameters of the GWB model can be fitted by using the measurement depression depth. The corresponding tangential velocity distribution of the vortex can be calculated accordingly. In their experiment results, the vortices with strong strength (the vortex Rossby number $\mathrm{Ro}_{v}$ is about 4.3) will produce weak Rossby wakes during their motion. The vortex with weaker strength (the vortex Rossby number $\mathrm{Ro}_{v}$ is about 1.8) maintains a clean single vortex structure during its movement. These phenomena are mainly related to the relative importance of the vortex $\beta$ effect and the planetary $\beta$ effect.

The present paper is the first of a series of works following Chen et al. [22] and is mainly devoted to validation of a proposed modified shallow water model (MSWM) by a joint experimental and numerical study. To improve the ability of capturing the flow features, a dissipative momentum flux term and an effective gravitation term were adopted in MSWM, while an artificial viscosity term was added to ensure numerical stability [23, 24]. We solved this modified shallow water model using a multidimensional positive definite advection transport algorithm (MPDATA) which was proposed and has been well known for simulations of geophysical flow by Smolarkiewicz and his colleagues for decades [25-28]. This study also proposes a theoretical analysis of dynamical similarity conditions to mimic the hurricane-like vortices on a $\beta$-plane by vortices generating in a rotating tank with a gently sloping bottom. The paper is organized as follows. First, Section 2 derived the dynamical similarity conditions for the model rotating tank experiment and the prototype hurricane-like motion by the potential vorticity conservation. Section 3 presents the numerical calculations of the strong cyclone motions in the rotating tank by a modified shallow water model. The simulation results by MSWM using the fitted parameters for gradient-wind-balance (GWB) model are shown in Section 4. We also investigate the long-term evolutions of strong cyclonic motion on different bottom slopes. Finally, conclusions are presented in Section 5.

\section{Governing Principle and Similarity Laws}

Let us now proceed with the governing principles of phenomena under consideration. Two important nondimensional parameters concerning the barotropic cyclonic motion must be first introduced. Let $V_{m}$ and $R_{m}$ be the characteristic velocity and length scales for cyclonic motion, respectively. We define the vortex Rossby number by

$$
\mathrm{Ro}_{v}=\frac{V_{m}}{f R_{m}},
$$

where $f$ is the planetary vorticity. The other is the vortex planetary $\beta$ parameter, defined by 


$$
\beta_{0}^{*}=\frac{\beta R_{m}^{2}}{V_{m}}
$$

where $\beta$ is planetary vorticity gradient. In this study, we are interested in those cyclonic motions which are $\mathrm{Ro}_{v} \sim O(1)$ and $\beta_{0}^{*} \sim O\left(10^{-2}-10^{-3}\right)$. In our terminology, such cyclones are called strong $\left(\mathrm{Ro}_{v} \sim \mathrm{O}(1)\right)$ and intense $\left(\beta_{0}^{*} \sim O\left(10^{-2}-10^{-3}\right)\right)$ cyclones. The cyclones are said to be strong because the characteristic relative vorticity $V_{m} / R_{m}$ of the cyclone is of the same order of magnitude with the planetary vorticity $f$. The cyclones are said to be intense because variation of the vorticity across the cyclone due to the relative vorticity gradient is much larger than that due to the planetary vorticity gradient in the vicinity of the cyclonic core structure [10]. For example, a tropical cyclone with moderate strength with a maximum tangential speed $V_{m}=40 \mathrm{~m} / \mathrm{s}$ and a corresponding radius $R_{m}=150 \mathrm{~km}$ results in $\operatorname{Ro}_{v} \sim 5.34$ and $\beta_{0}^{*} \sim 0.0121$.

For a rotating, shallow water flow, the governing principle is the law of conservation of potential vorticity (PV) [29].

$$
\frac{D \Pi}{D t}=0
$$

where potential vorticity $\Pi$ is defined as $\Pi=(f+\zeta) / H$ where $H$ is the fluid layer depth.

2.1. PV Conservation for the Model Problem. As shown in Figure 1(a), fluid layer depth $H(x, y, t)$ can be expressed as $H=H_{0}+\eta-h_{B}$, where $H_{0}$ is the unperturbed depth, $\eta(x, y, t)$ is the free-surface deviation due to the motion, and $h_{B}(x, y)$ represents the bottom topography. The local Cartesian coordinates $x, y$, and $z$ point horizontally inward, westward, and vertically upward, respectively. We can choose a gentle-slope (denoted as $s_{y}$ ) bottom topography in a rotating fluid confined in a tank spinning at a constant speed $\Omega=f_{0} / 2$, where $f_{0}$ is the background vorticity of the rotating fluid. The flow dynamical features in the tank experiment can be understood by the law of conservation of potential vorticity (PV) by assuming small variations of $\eta$ and $h_{B}=s_{y} y$ with respect to $H_{0}$; that is, $\eta / H_{0} \ll 1$, $s_{y} y / H_{0} \ll 1$. This allows (3) to be written as

$$
\frac{D}{D t}\left(\frac{f_{0}}{H_{0}}\left(1-\frac{\eta}{H_{0}}+\frac{s_{y} y}{H_{0}}\right)+\frac{\zeta}{H_{0}}\left(1-\frac{\eta}{H_{0}}+\frac{s_{y} y}{H_{0}}\right)\right)=0 \text {. }
$$

Next, we will give an appropriate choice on scaling the cyclonic motion so that the magnitudes of the nondimensional variables are of order unity. Take the maximum tangential speed of the cyclone $V_{m}$ as the reference velocity, $\zeta_{m}=V_{m} / R_{m}$ as the reference vorticity, and $\zeta_{m}^{-1}$ as the reference time (the vortex turnaround time). In addition, we choose the maximum vortex depression $\eta_{v}$ as the reference free-surface deviation, and thus we have the set of nondimensional variables defined by $t^{*}=t / \zeta_{m}^{-1}, y^{*}=y / R_{m}, \eta^{*}=\eta / \eta_{v}$, and $\zeta^{*}=\zeta / \zeta_{m}$. Since $f_{0} / H_{0}$ is constant and makes no contribution, we can divide equation (4) by $\zeta_{m}^{2} / H_{0}$ to obtain

$$
\frac{D}{D t^{*}}\left(\beta_{y}^{*} y^{*}-\beta_{v}^{*} \eta^{*}+\zeta^{*}\left(1+s_{y}^{*} y^{*}-s_{v}^{*} \eta^{*}\right)\right)=0 .
$$

Equation (5) is a nondimensional PV conservation law of a strong cyclonic vortex translating on a sloping bottom in a rotating tank. There are four nondimensional parameters involved from different sources of layer depth variation. They are (i) the bottom slope parameter $s_{y}^{*}=s_{y} R_{m} / H_{0}$, where $s_{y}$ represents the bottom slope, and (ii) the bottom $\beta$-parameter,

$$
\beta_{y}^{*}=\frac{f_{0} s_{y} R_{m}^{2}}{V_{m} H_{0}}=\frac{s_{y}^{*}}{\mathrm{Ro}_{v}},
$$

(iii) the vortex slope parameter $s_{v}^{*}=s_{v} R_{m} / H_{0}$, where a characteristic vortex slope is defined as $s_{v}=\eta_{v} / R_{m}$, and (iv) the vortex $\beta$-parameter

$$
\beta_{v}^{*}=\frac{f_{0} s_{v} R_{m}^{2}}{V_{m} H_{0}}=\frac{s_{v}^{*}}{\mathrm{Ro}_{v}} .
$$

The physical meaning of (5) can be understood by applying it far away from the cyclonic structure where both the nondimensional relative vorticity and surface depression are small; that is, $\zeta^{*} \sim O(\varepsilon), \eta^{*} \sim O(\varepsilon)$. Then, we have

$$
\frac{D}{D t^{*}}\left(\beta_{y}^{*} y^{*}+\zeta^{*}\right)=0 \text {. }
$$

Equation (8) is the traditional PV conservation law which explains the generation of Rossby wave in a depthvarying fluid layer. On the other hand, we can rearrange (5) as

$$
\frac{D}{D t^{*}}\left(\beta_{y}^{*} y^{*}+\left(\zeta_{e}^{*}\right)_{t}\right)=0,
$$

where $\left(\zeta_{e}^{*}\right)_{t}$ is the equivalent relative vorticity, defined by

$$
\left(\zeta_{e}^{*}\right)_{t}=\zeta^{*}\left(1+s_{y}^{*} y^{*}-s_{v}^{*} \eta^{*}\right)-\beta_{v}^{*} \eta^{*}
$$

Here, the subscript $t$ denotes a tank experiment. Equations (9) and (10) state that the strong cyclonic motion moving towards the shallower region will decrease their equivalent relative vorticity.

2.2. PV Conservation for Prototypical Problem. As shown in Figure 1(b), we now consider the potential vorticity conservation for strong cyclonic motion on a $\beta$-plane with a constant layer depth. The planetary vorticity $f$ can be linearized by the $\beta$-plane approximation; that is, $f \sim f_{0}+\beta_{0} y$, where $f_{0}$ and $\beta_{0}$ are constants, and $y$ is the local northward Cartesian coordinate. Considering small relative variation of $\eta$ and assuming that no bottom topography is present, that is, $\eta / H_{0} \ll 1, h_{B} \approx 0$, we can rewrite (4) as

$$
\frac{D}{D t}\left(\frac{f_{0}}{H_{0}}\left(1-\frac{\eta}{H_{0}}\right)+\frac{\beta_{0} y}{H_{0}}+\frac{\zeta}{H_{0}}\left(1-\frac{\eta}{H_{0}}\right)\right)=0,
$$

for a strong cyclonic vortex. Next, we will give an appropriate choice on scaling the cyclone motion so that the magnitudes of the nondimensional variables are of order 


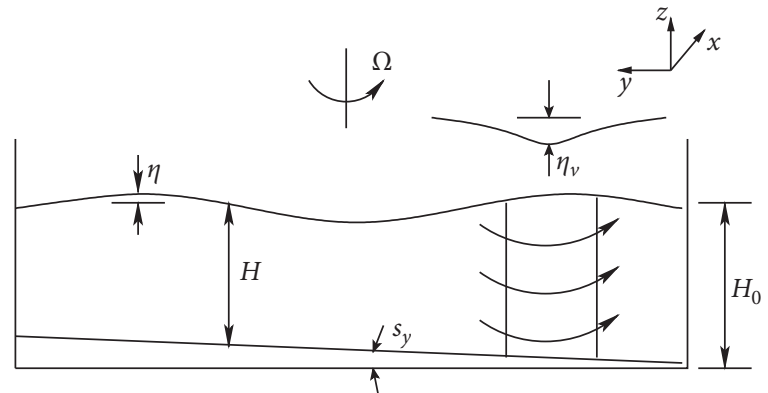

(a)

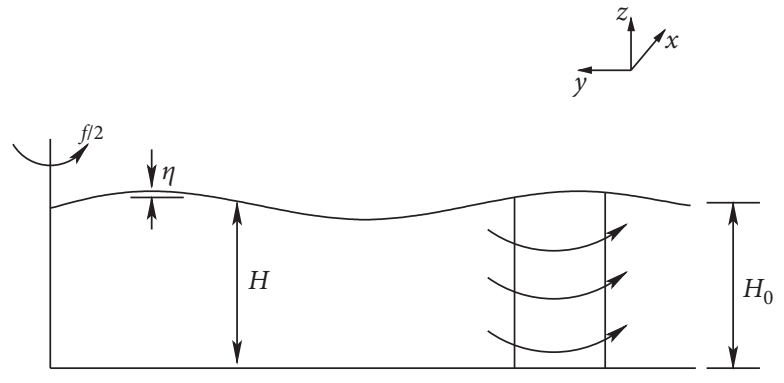

(b)

Figure 1: (a) Schematic of a cyclonic vortex motion in a rotating tank with a sloping bottom. (b) Schematic of a cyclonic motion on a $\beta$-plane.

unity. The reference scales are taken identically as those in the tank experiment. Since $f_{0} / H_{0}$ is constant and makes no contribution, we can divide (11) by $\zeta_{m}^{2} / H_{0}$ and yield

$$
\frac{D}{D t^{*}}\left(-\beta_{v}^{*} \eta^{*}+\beta_{0}^{*} y^{*}+\zeta^{*}\left(1-s_{v}^{*} \eta^{*}\right)\right)=0
$$

where the nondimensional variables are defined the same as in the rotating tank experiment. Equation (12) is a nondimensional PV conservation law of strong cyclonic motions translating on a $\beta$-plane with no topographic feature.

Notably, (12) bears a close physical explanation on the potential vorticity dynamics as (5). That is, when the fluid particles move northward (with higher $f$ ), the relative vorticity decreases and the surface depression varies simultaneously. We can also define an equivalent relative vorticity for the prototype flow field as

$$
\left(\zeta_{e}^{*}\right)_{f}=\zeta^{*}\left(1-s_{v}^{*} \eta^{*}\right)-\beta_{v}^{*} \eta^{*}
$$

Although the explanations of the change of equivalent relative vorticity of (5) and (12) are very similar, the mutual adjustment of the relative vorticity and the surface depression of the vortex in the model experiment is more complicated than that in the prototypical flow. An additional $\operatorname{term} \zeta^{*}\left(s_{y}^{*} y^{*}\right)$ is involved in (10) and this makes it difficult to decouple the effects contributed by the northward movement or the surface depression for the change of relative vorticity in the strong cyclonic motion.

2.3. Dynamical Similarity Conditions. In order to derive the dynamical similarity conditions, we first apply (5) and (12) far away from the cyclonic core region where the nondimensional quantities $\zeta^{*}$ and $\eta^{*}$ are small. Comparing those terms on the left-hand sides of (5) and (12), we have the first similarity condition

$$
\beta_{y}^{*}=\beta_{0}^{*}
$$

Then, we apply (5) and (12) in the core region of cyclonic structure with $\zeta^{*} \approx O(1)$. By evaluating both equations on the specific locations that yield unity $\left(\zeta^{*}\right)_{m}$ and $\left(\zeta^{*}\right)_{p}$, where the subscripts $m$ and $p$ denote, respectively, the model (rotating tank) and prototypical (field) experiment, also, we assume that the cyclonic vortex is generated on the initial latitude, that is, $\left(y^{*}\right)_{m} \sim 0$ and $\left(y^{*}\right)_{p} \sim 0$. Therefore, comparing those terms on the left-hand sides of (5) and (12), we obtain the second similarity condition

$$
\left(\left(\operatorname{Ro}^{-1}+1\right) s_{v}^{*}\right)_{m}=\left(\left(\operatorname{Ro}^{-1}+1\right) s_{v}^{*}\right)_{p} .
$$

Equations (14) and (15) build a dynamical connection from model experiment and the prototypical flow by sharing the similar dynamics according to the conservation of potential vorticity.

\section{Numerical Calculations}

3.1. Modified Shallow Water Model. As shown in Figure 1(a), let $\vec{u}=(u, v)$ be the horizontal velocity, let $H$ be the fluid layer depth, and let $h_{B}$ be the elevation of the bottom topography, respectively. In order to model the viscous friction in the rotating shallow water flow, the present study incorporates an additional term $\nu \nabla \cdot \nabla \vec{u}$ in the standard shallow water model as suggested in $[23,24]$.

$$
\begin{gathered}
\frac{\partial H}{\partial t}+\nabla \cdot(\vec{u} H)=0, \\
\frac{D \vec{u}}{D t}+g_{e} \nabla\left(h_{B}+H\right)+f \vec{k} \times \vec{u}=\nu \nabla \cdot \nabla \vec{u}
\end{gathered}
$$

where the effective gravity $g_{e}$ is defined as [30]

$$
g_{e}=\sqrt{g^{2}+\frac{f^{4} \rho_{r}^{2}}{4}} .
$$

In (18), $g_{e}$ is defined as the net acceleration directing perpendicular to the free surface and $\rho_{r}$ is the distance of the fluid particle to the rotation axis; and $v$ denotes the kinematic viscosity coefficient of the working fluid (in the present study, $v=1 \times 10^{-6} \mathrm{~m}^{2} \mathrm{~s}^{-1}$ for water at $\left.20^{\circ}\right)$. By choosing the same set of reference scales of the vortex motion as in the last section, we can recast (16) and (17) into the following modified shallow water model in flux form: 


$$
\begin{gathered}
\frac{\partial H^{*}}{\partial t^{*}}+\nabla^{*} \cdot\left(\vec{u}^{*} H^{*}\right)=\widehat{v}_{H} \nabla^{*} \cdot \nabla^{*} H^{*} \\
\frac{\partial H^{*} \vec{u}^{*}}{\partial t^{*}}+\nabla^{*} \cdot\left(\vec{u}^{*} H^{*} \vec{u}^{*}\right)=-\operatorname{Ro}_{v}^{-1} \vec{k} \times\left(H^{*} \vec{u}^{*}\right) \\
\quad-F^{-2} H^{*} \nabla^{*}\left(h_{B}^{*}+H^{*}\right)+\operatorname{Re}^{-1} \nabla^{*} \cdot H^{*} \nabla^{*} \vec{u}^{*}
\end{gathered}
$$

In (19), an artificial viscosity term is incorporated to ensure the numerical stability. It is defined as $\widehat{v}_{H}=1 \times 10^{-8} \Delta t^{-1} \Delta x$, where $\Delta t$ and $\Delta x$ are time increment and mesh spacing, respectively. It is noted that, in (20), there are three nondimensional parameters. The vortex Rossby number is defined in (1), the vortex Froude number is defined as $\mathrm{Fr}=V_{m} / \sqrt{g_{e} H_{0}}$, and the vortex Reynolds number is defined by $\operatorname{Re}=R_{m} V_{m} / \nu$. The transport momentum variables $U_{i, j}:=\left(H^{*} u^{*}\right)_{i, j}$ and $V_{i, j}:=\left(H^{*} v^{*}\right)_{i, j}$ and layer-depth $H_{i, j}^{*}$ are given on a rectangular grid, while the advective velocity components $u_{i+1 / 2, j}$ and $v_{i, j+1 / 2}$ are staggered by a one-half grid spacing. Here $(i, j)$ denotes the location in the grid and $\vec{V}=(U, V)$ denotes the momentum variable. The MSWM were discretized on an Arakawa-C staggered grid. The forcing terms in MSWM are discretized by following the suggestions in the works of Schär and Smith [23, 24].

3.2. MPDATA Scheme. The discretized equations of the MSWM were solved by the MPDATA (multidimensional positive definite advection transport algorithm) which was proposed by Smolarkiewicz and his colleagues [25-28]. MPDATA is a procedure that iteratively approximates the advection equation, which uses a donor cell approximation to compensate the truncation error of the original donor cell scheme. This step may be repeated an arbitrary number of times, leading to successively more accurate solutions of the advection equation. Concerning the contributions of forcing terms on the transport variables, we have incorporated the MPDATA scheme by a Strang-splitting method and to implement the predictor-corrector concept for ensuring the time marching accuracy to second order. The transport variables $\psi_{i, j}^{n+1}$ at time level $n+1$ can evaluated by MPDATA scheme by incorporating the contributions from the forcing terms

$$
\psi_{i, j}^{n+1}=\operatorname{MPDATA}\left(\psi_{i, j}^{n+1}+0.5 \Delta t R_{i, j}^{n}, \vec{u}_{i+(1 / 2)}^{n+1 / 2} \vec{e}_{j}\right)+0.5 \Delta t R_{i, j}^{n+1},
$$

where $\psi_{i, j}^{n+1}$ are the transport variables, $R_{i, j}^{n+1}$ are the forcing terms, and $\Delta t$ is the time increment. In the above equation, MPDATA symbolizes the homogeneous transport algorithm. Advecting the auxiliary field $\psi_{i, j}^{n}+0.5 \Delta t R_{i, j}^{n}$ not only compensates the truncation error due to the forcing terms but also has the physical interpretation of integrating the forces along a parcel trajectory rather than at the grid point. This makes (21) congruent to semi-Lagrangian approximations and facilitates unified fluid models that integrate the equations of motion, optionally, in the Eulerian (point-wise) or Lagrangian (trajectory-wise) sense.
3.3. Boundary Conditions Treatment. On the treatment of boundary conditions, the relaxation boundary concept proposed by Davies is used [31]. The eight grid points nearest to the lateral boundary are a dedicated relaxation zone in which the height and momentum field are relaxed towards the externally specified unperturbed values after every time step. The relaxation coefficients are chosen as 1.0, 0.98, 0.9, $0.75,0.5,0.25,0.1$, and 0.02 .

\section{Results and Discussion}

4.1. Initial Vortex Structure. In the present study, we follow the approach in the previous study [22] to identify and to simulate the strong cyclonic motions using the gradientwind-balance vortex distribution

$$
\begin{aligned}
& v=-\frac{f r}{2}+\sqrt{\frac{f^{2} r^{2}}{4}+g A B \eta_{\nu} \exp \frac{\left(-A r^{-B}\right)}{r^{B}}}, \\
& h=H_{0}-\eta_{v}\left(1-\exp \left(-A r^{-B}\right)\right),
\end{aligned}
$$

where $A$ and $B$ are, respectively, the vortex size parameter and the vortex shape parameter. Equations (22) and (23) are derived from an analytic model for radial profiles of sea level pressure and winds in a hurricane which was proposed by Holland [32]. In addition, owing to the velocity distribution of the GWB vortex model being assumed to be axisymmetric, the estimated vertical component of vorticity $\widehat{\zeta}$ can be derived by

$$
\widehat{\zeta}=\frac{1}{\widehat{r}} \frac{\partial \widehat{r} v_{\theta}}{\partial \widehat{r}}
$$

Therefore, the estimated vorticity distribution can be expressed as

$$
\begin{aligned}
\widehat{\zeta} & =-f+\frac{1}{2}\left(\frac{f^{2} \widehat{r}^{-4}}{4}+g A B \eta_{v} \widehat{r}^{2-B} e^{-A \widehat{r}^{-B}}\right)^{-1 / 2} \\
& \times\left(f^{2} \widehat{r}^{-2}+g A B \eta_{v}\left(2-B+A B \widehat{r}^{-B}\right) \widehat{r}^{-B} e^{-A \widehat{r}^{-B}}\right) .
\end{aligned}
$$

In [22], two laboratory vortices $S$ and $W$ were generated by different strengths. $S$ was created to be a larger depression by a rotating oar than $W$ created by a rotating solid cylinder. Figures 2(a) and 2(b) display the fitted results of the vortex depression and the azimuthal velocity distribution of $S$ and $W$, respectively. It is noted that the measurements of surface depression enable the vortex structure to be fitted with satisfactory confidence. The error bar of the depression measurement is approximately $0.001 \mathrm{~cm}$. The vortex size parameters $A$ for vortices $S$ and $W$ are approximately 2.59 and 2.48 , respectively. The vortex shape parameters $B$ for vortices $S$ and $W$ are approximately 1.24 and 0.89 , respectively.

Figure 2(c) illustrates the distributions of the estimated vorticity $\widehat{\zeta}$ in (25) of vortices $S$ and $W$ as measured in [22]. The results show that vortices $S$ and $W$ are both vortices of large Rossby number, while the maximum value of $\widehat{\zeta}$ for vortex $S$ was approximately 15.70 , which was about twice 


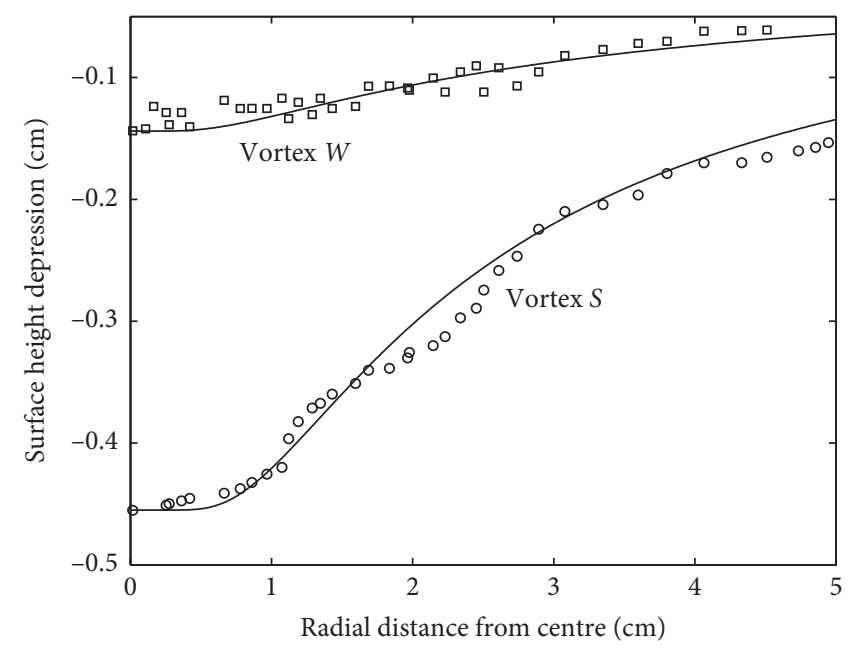

(a)

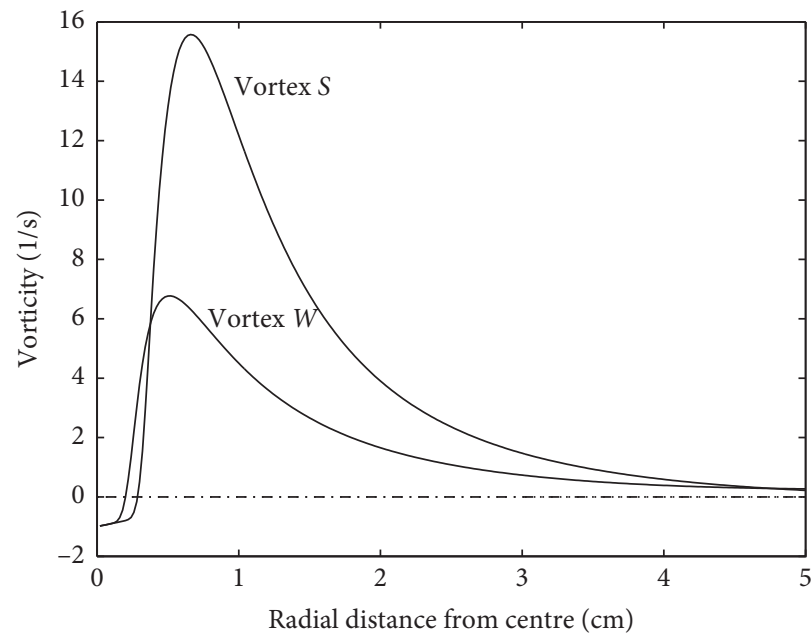

(c)

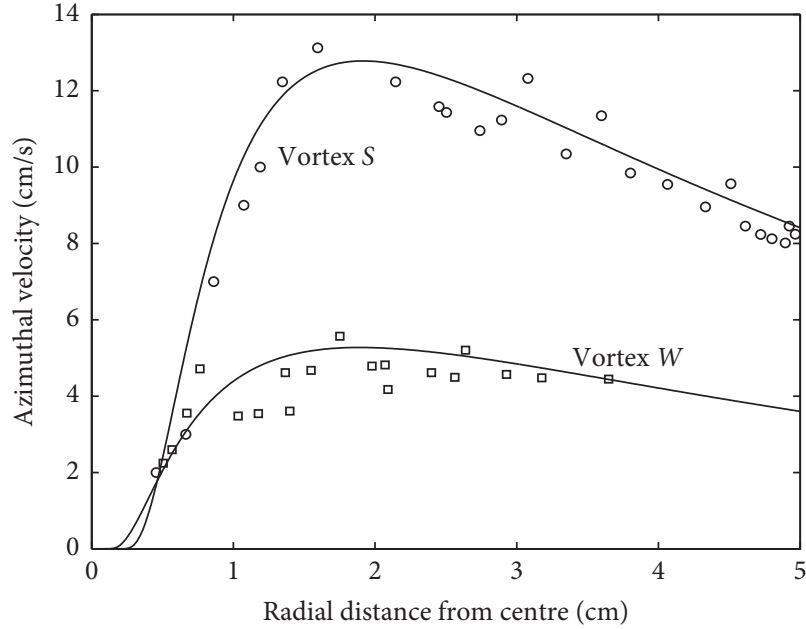

(b)

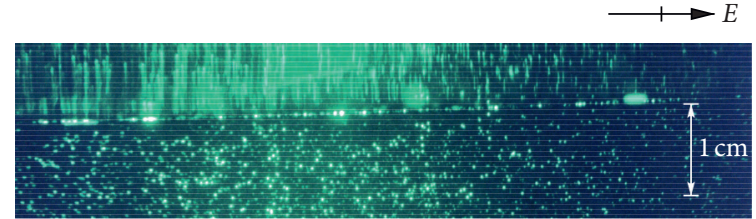

(d)

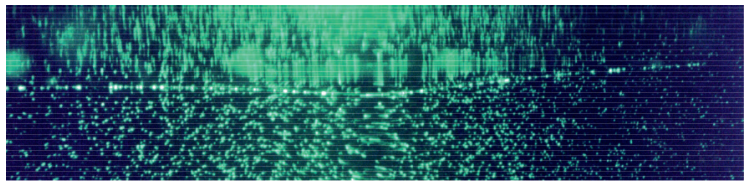

(e)

FIgURE 2: The GWB fitted distributions of cyclonic vortices $S$ and $W$ of (a) surface depression, (b) azimuthal velocity distribution, and (c) vorticity distribution in a radial direction. The circles represent the measured values of vortex $S$ and the square symbols show the measured values of vortex $W$. Photographs showing the difference of surface profile at (d) the reference snapshot and (e) the snapshot of vortex centre.

that of the vortex $W$ (approximately 6.75). Figure 2(d) shows a reference photograph of a surface profile illuminated by a vertical light sheet taken from the south end of the tank to the north. Figure 2(e) shows a picture of the surface profile of the vortex centre passing through a vertical light sheet. Comparing Figure 2(e) with Figure 2(d), we can extract the actual surface depression depth of the vortex centre.

4.2. Time Evolutions and Structure Change of Vortices $S$ and $W$. Figures 3 illustrates the calculation results of the relative vorticity of the vortex $S$ by MSWM on a sloping bottom in a rotating tank. The domain of numerical calculation is
$120 \mathrm{~cm} \times 120 \mathrm{~cm}$ on a $600 \times 600$ uniform rectangular grid system. The vortex was generated about $45 \mathrm{~cm}$ and $40 \mathrm{~cm}$ away from the south and the east tank boundaries, respectively, to relax the boundary effect. Compared with the experimental results in the previous study [22], Figures 4 and 5 show a qualitatively close agreement between the experimental and numerical approaches of the vortex $W$. Fairly symmetric isolated vortices were observed experimentally and numerically during their northwest drifts on a sloping bottom. Outside the primary vortex structure, there exists a weak anticyclonic vorticity patch. This flow feature is gradually induced by the Rossby wave radiation accompanied by the primary vortex as indicated in [10] and is observed both in the 


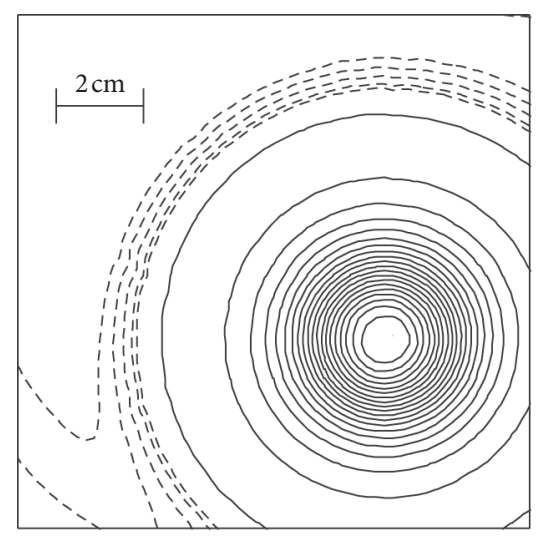

(a)

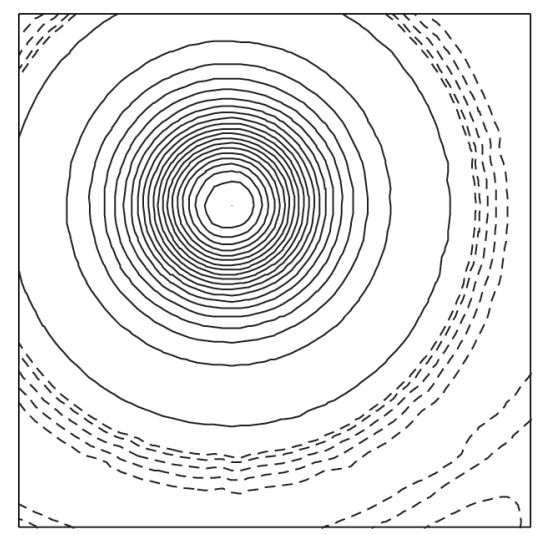

(d)

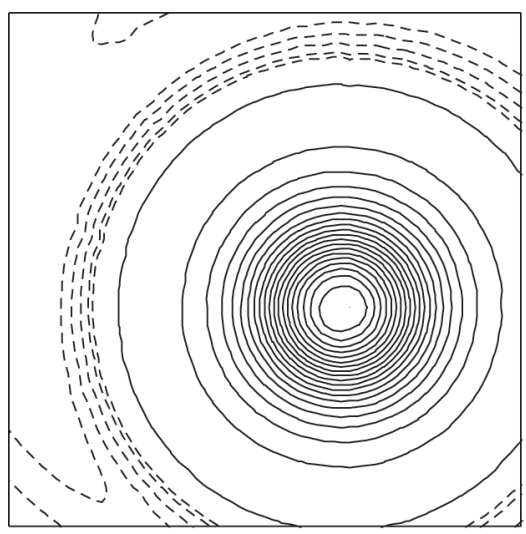

(b)

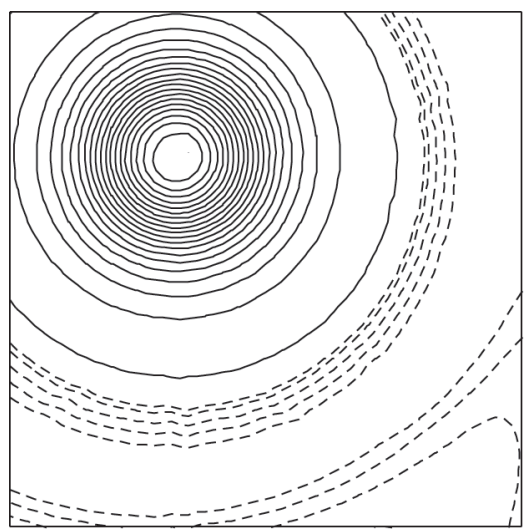

(e)

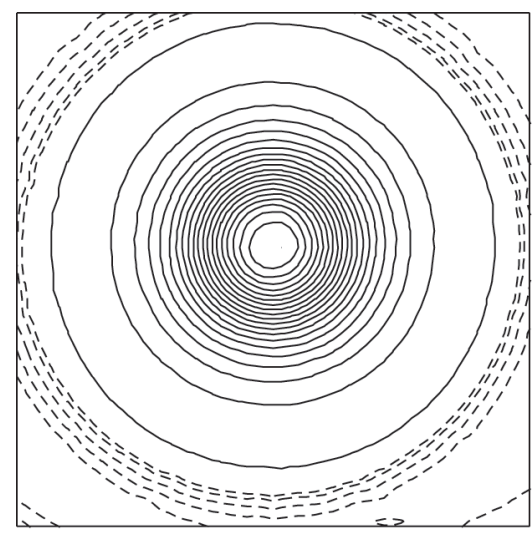

(c)

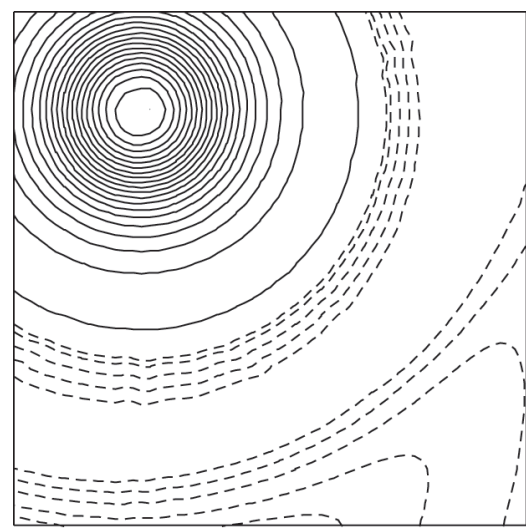

(f)

FIgURE 3: Computational results showing the evolution of the relative vorticity of vortex $S$ at the time instances (a) $5 \mathrm{~s}$, (b) $9 \mathrm{~s}$, (c) $11 \mathrm{~s}$, (d) $13 \mathrm{~s}$, (e) $15 \mathrm{~s}$, and (f) $17 \mathrm{~s}$ after the oar was lifted. The size of the window is about $12 \mathrm{~cm}$ square area. The solid lines represent the positive vorticity, while the dashed lines indicate the negative vorticity.
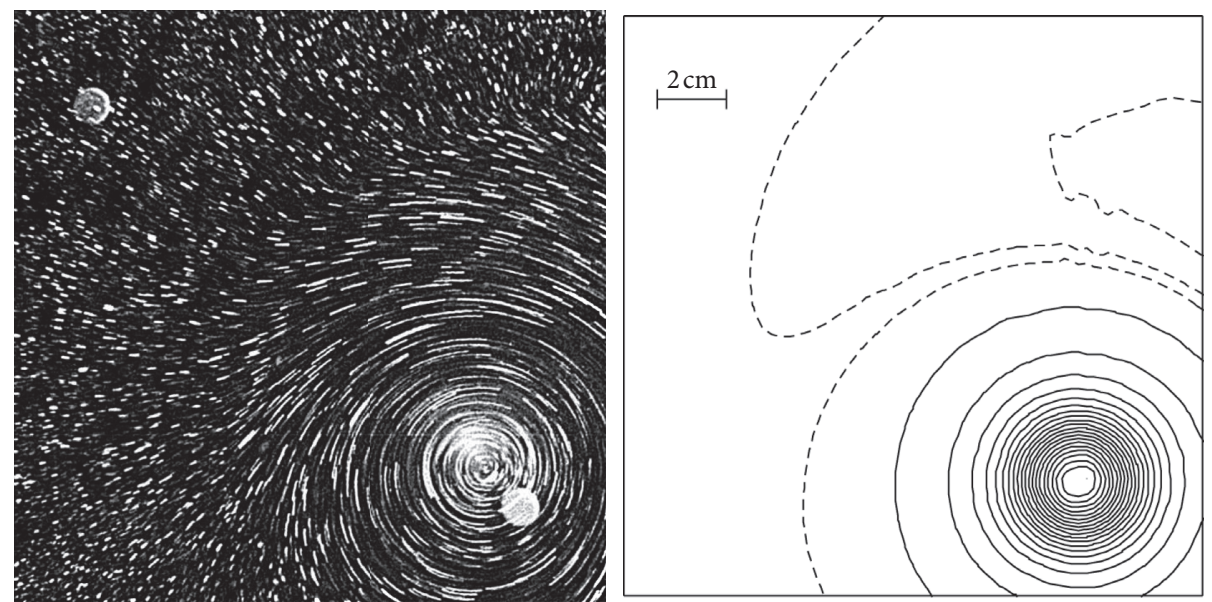

(a)

Figure 4: Continued. 

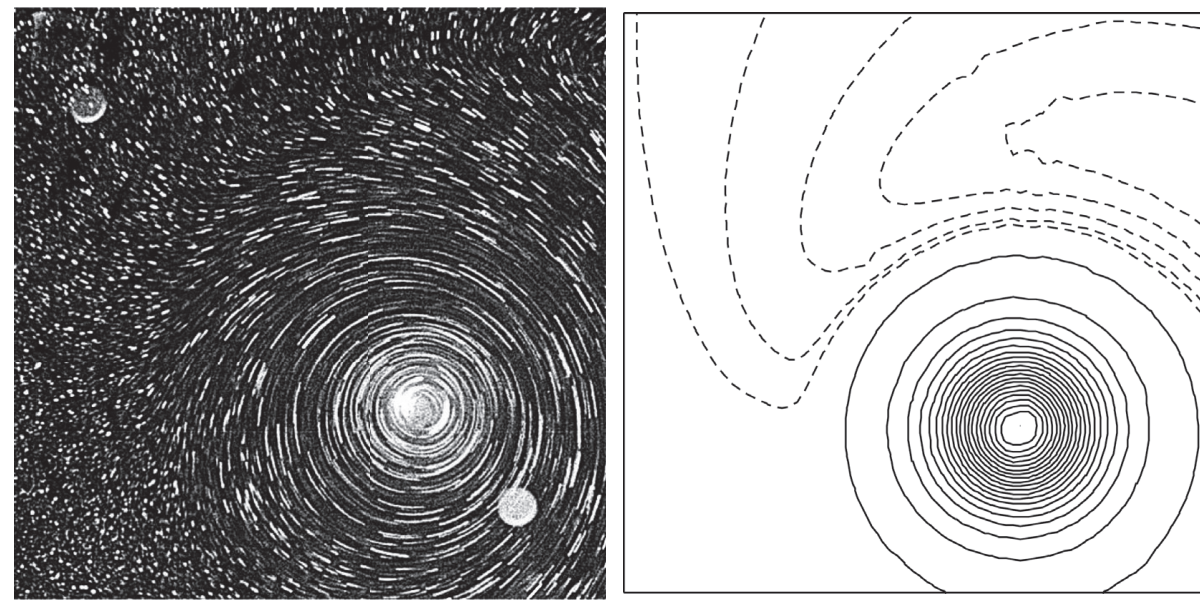

(b)
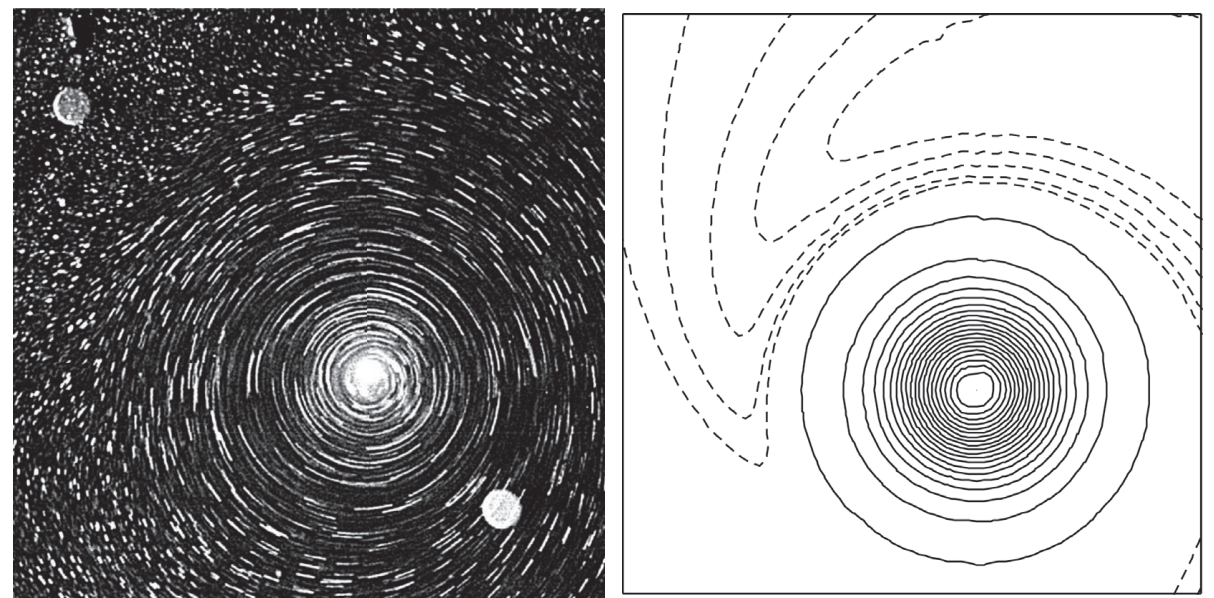

(c)

FIGURE 4: Comparison of experiment and numerical results for a stirred vortex $W$ at the time instances (a) $6 \mathrm{~s}$, (b) $10 \mathrm{~s}$, and (c) $14 \mathrm{~s}$ after the cylinder was lifted. The size of the window is about $17 \mathrm{~cm}$ square area. Note that the experiment photographs are adapted from [22] for comparison with numerical results.
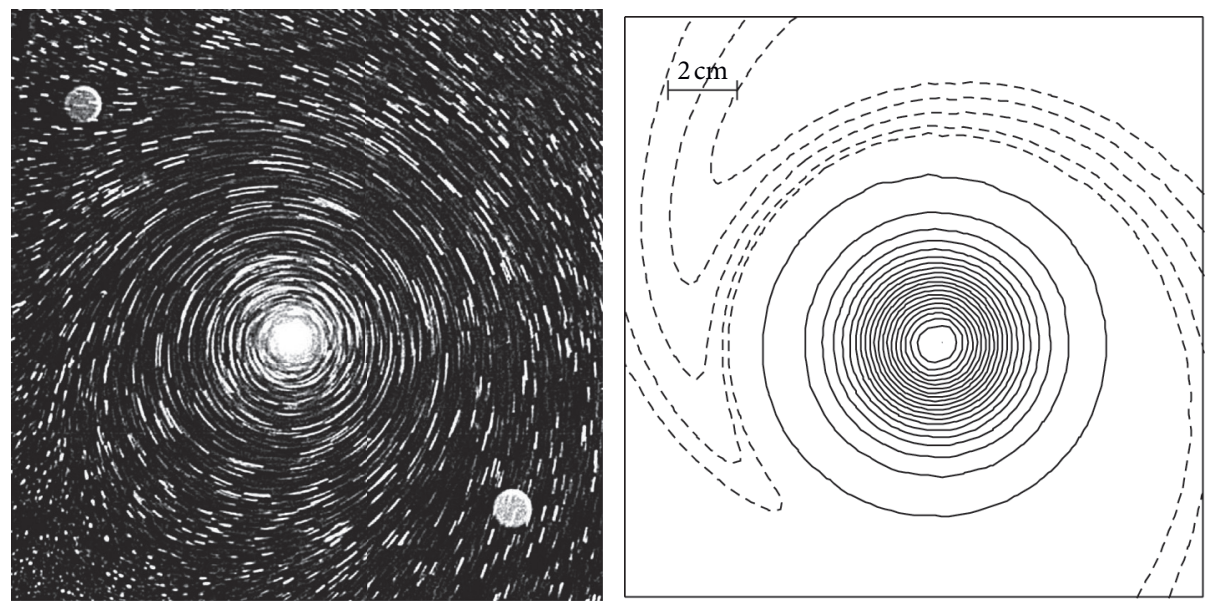

(a)

Figure 5: Continued. 

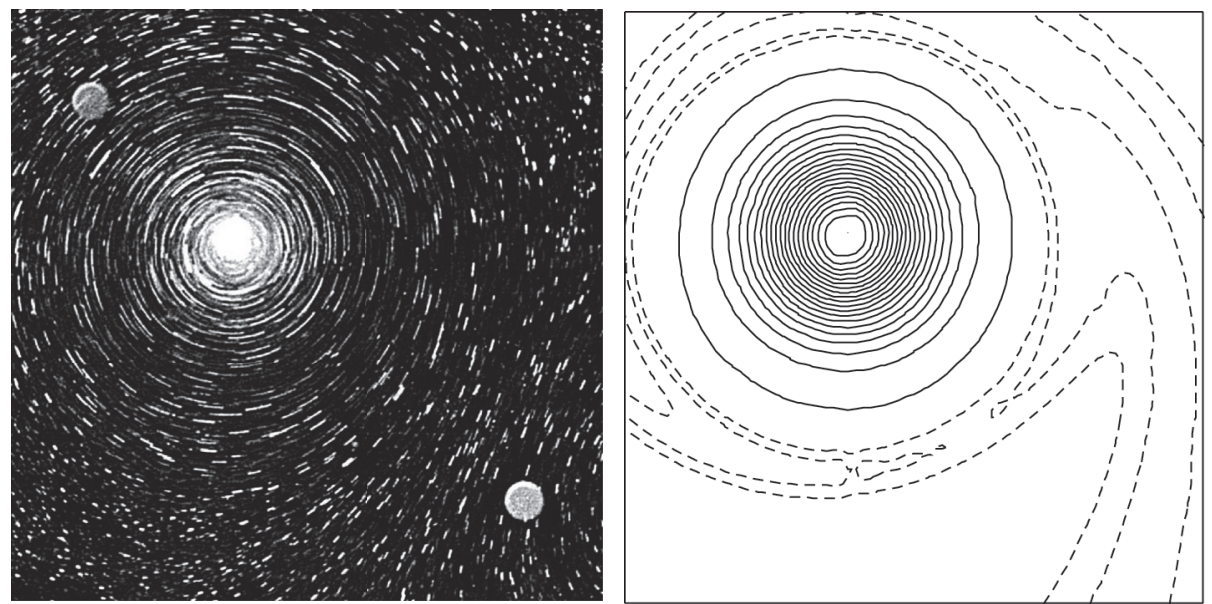

(b)
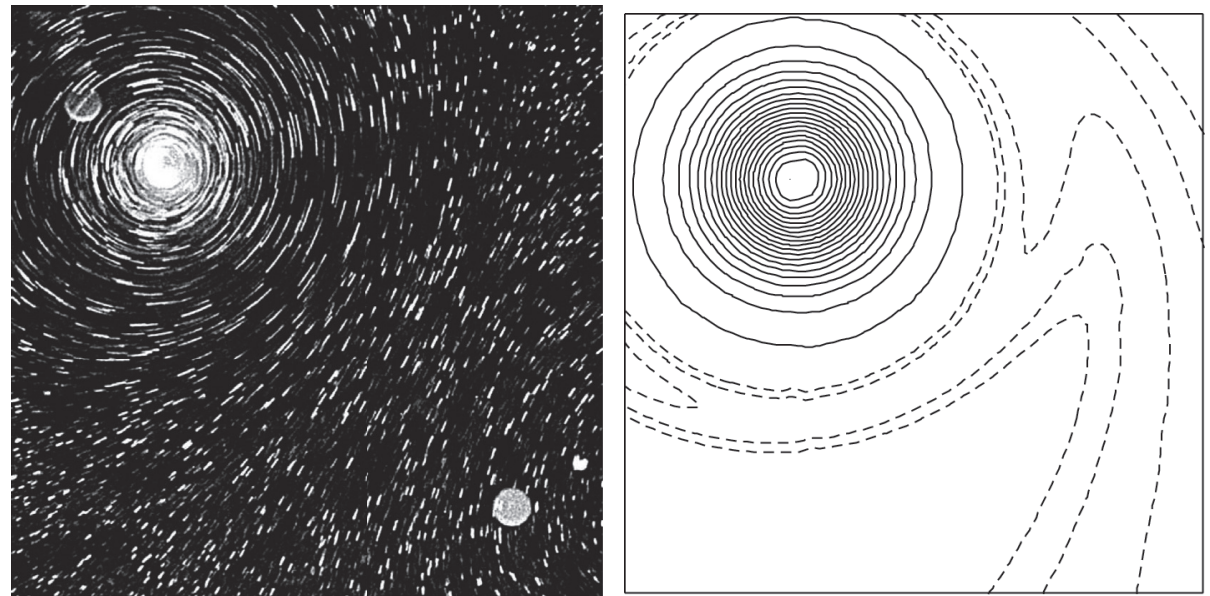

(c)

Figure 5: Similar to Figure 4 but at the time instances (a) $18 \mathrm{~s}$, (b) $22 \mathrm{~s}$, and (c) $28 \mathrm{~s}$ after the cylinder was lifted. The size of the window is about $17 \mathrm{~cm}$ square area.

experiments and in the numerical simulations. It is noted that the ratio of the vortex $\beta$ effect and the planetary $\beta$ effect $\gamma=$ $\beta_{v}^{*} / \beta_{y}^{*}$ reflects the nonlinearity against the linear Rossby wave radiation. In this study, the value of $\gamma$ corresponding to vortex $S$ and vortex $W$ is about 4.437 and 1.385 , respectively. It can be seen that the ability of vortex $S$ to resist Rossby wave radiation is better than that of vortex $W$.

Figure 6 demonstrates the evolution of vortex structures of vortex $W$ at (a) $t=10 \mathrm{~s}$ and (b) $t=28 \mathrm{~s}$ after the cylinder was lifted. The solid lines denote the radial distribution of the vertical component of vorticity, the dash-dotted lines indicate the radial distribution of the azimuthal velocity, and the dashed lines show the radial distribution of the surface depression. Notably, these radial distributions of vortex structure are obtained at a cross-sectional plane passing through the vortex with maximum depression. For convenience, only the values at the east of the vortex are plotted. The nondimensional relative vorticity shows a gradually decreasing tendency of its peak value at the vortex central region. The vortex distribution becomes smoother or remains constant at later times as long as the vortex travels to the northwest.
Additionally, the distribution of the azimuthal velocity shows that the maximum value approximately remains constant, while its corresponding radius gradually becomes larger than the initial radius $R_{m}$. Finally, the distribution of the vortex depression also displays a significant decreasing tendency when the vortex evolves to the northwest.

4.3. Vortex Trajectory and Intensity Change. Regarding the vortex trajectories, this study used the circle and square symbols in Figure 7 to indicate the measured tracks of vortex $S$ and vortex $W$, respectively. The solid lines represent the associated MSWM calculated vortex tracks. The dashed lines show that the contour levels of the topographic features $h_{B}^{*}$ calculated from south to north are approximately 0.387 , $0.401,0.415,0.429$, and 0.433 , respectively. The nondimensional topographic heights $h_{B}^{*}$ consist of the topographic sloping bottom $h_{s}^{*}$ and free surface deformation $h_{p}^{*}$ owing to the tank rotation as mentioned in [22]. It is worth noting that, compared with the contribution of inclined bottom deformation $h_{s}^{*}$ to the northwest drift of the vortex, the 


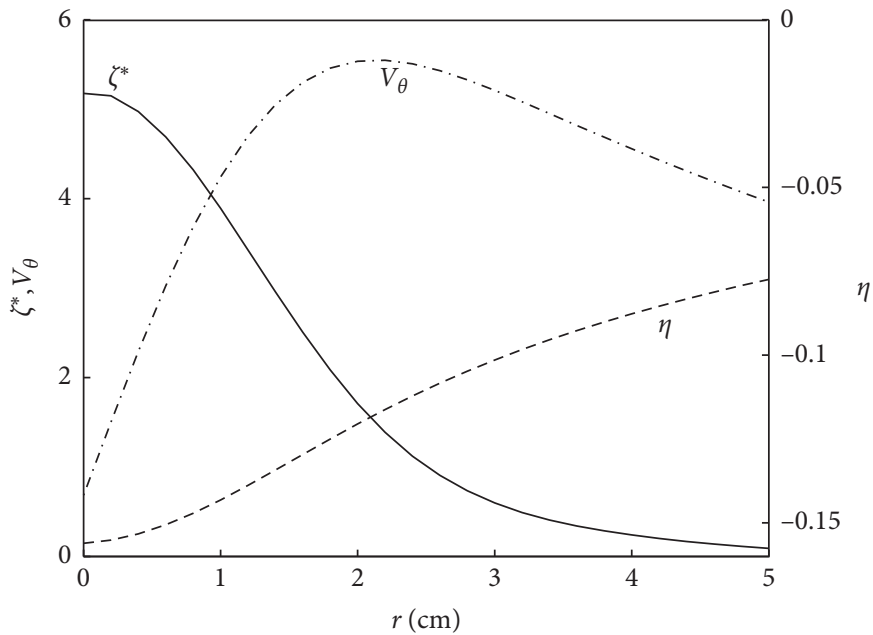

(a)

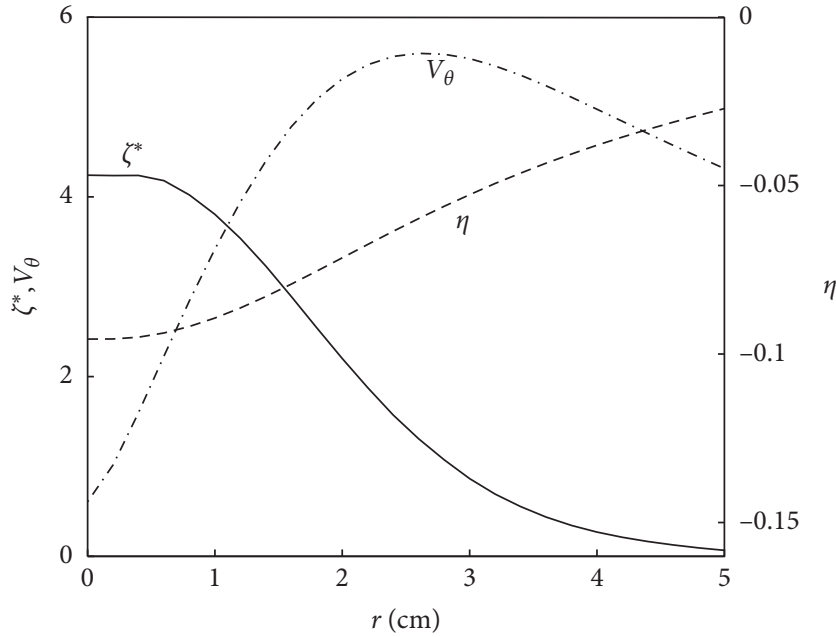

(b)

Figure 6: Evolution of structures of vortex $W$ at (a) $t=10 \mathrm{~s}$ and (b) $t=28 \mathrm{~s}$ after the cylinder was lifted.

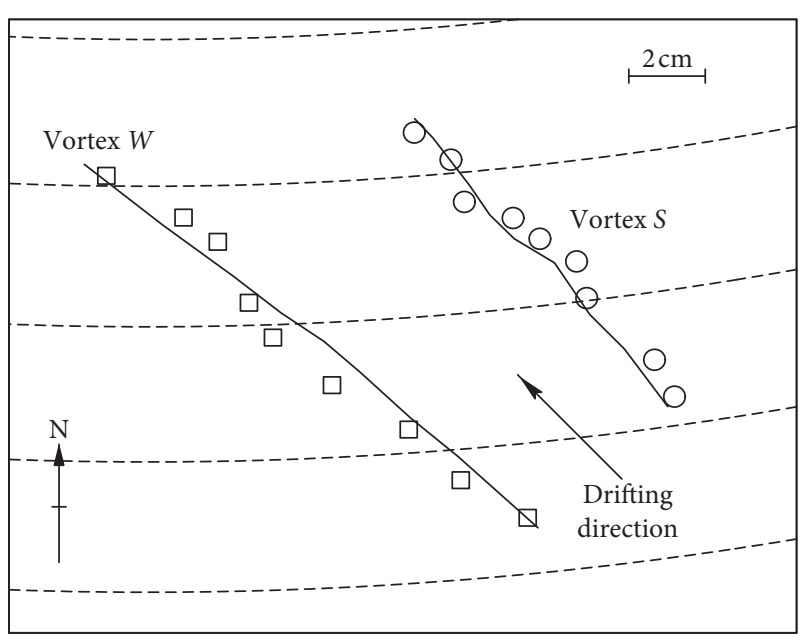

FIGURE 7: Graph showing the trajectories of the cyclonic vortices $S$ and $W$. contribution of free surface deformation $h_{p}^{*}$ to the northwest drift of the vortex is much smaller.

Figure 8(a) monitors the time variations of the maximum value of the azimuthal velocity $V_{\theta \text {, max }}$ as intensity change of vortex. They are recorded by the numerical simulation (denoted as a solid line) or the streak photography (represented as circles). The results show that the variations of $V_{\theta, \max }$ obtained from these two approaches displayed good agreement. It is noted that the calculated $V_{\theta, \max }$ decayed from its initial value $12.43 \mathrm{~cm} / \mathrm{s}$ to $9.82 \mathrm{~cm} / \mathrm{s}$ in 15 seconds. In the laboratory, $V_{\theta, \max }$ was estimated as decaying from $12.92 \mathrm{~cm} / \mathrm{s}$ to $9.79 \mathrm{~cm} / \mathrm{s}$. Figure 8 (b) shows the time variations of the maximum vorticity $\zeta_{\max }$ that is approximated by assuming that the vortex is axis-symmetric. The measurement results revealed good agreement of the maximum values of azimuthal velocity and vertical component of vorticity between these two approaches. 


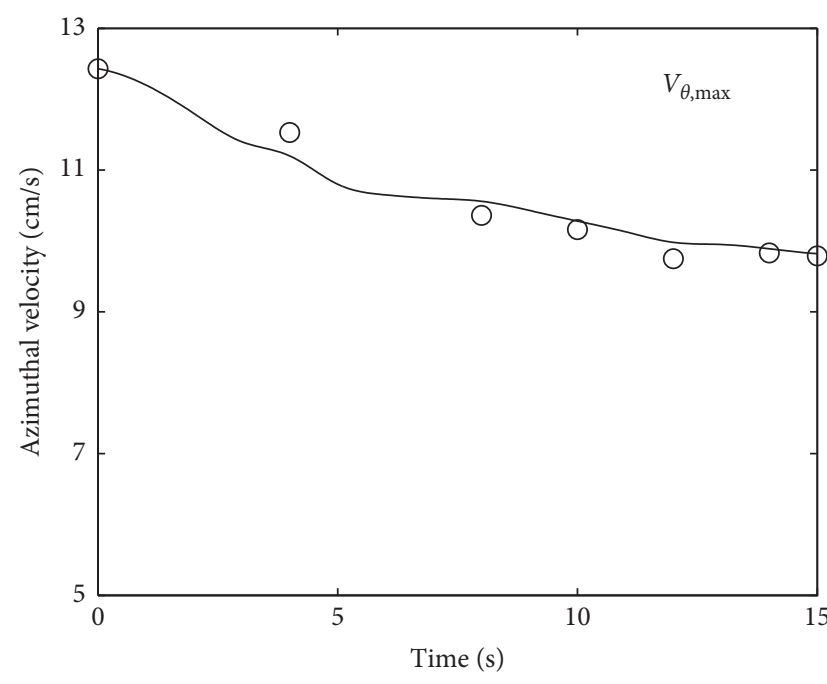

(a)

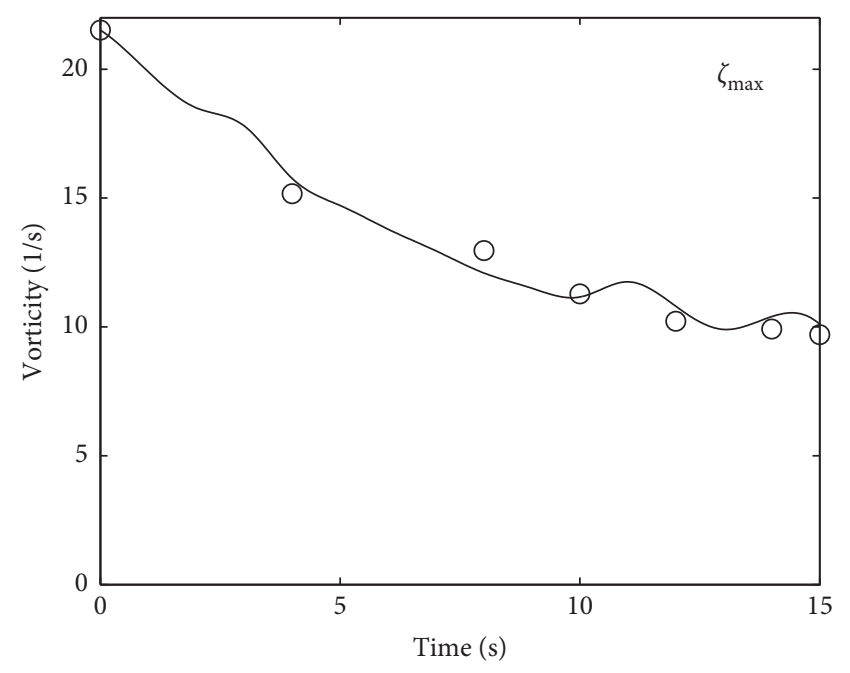

(b)

Figure 8: Time variations of (a) the maximum azimuthal velocity $V_{\theta, \max }$ and (b) the maximum vorticity $\zeta_{\max }$ of vortex $S$.

4.4. Effect of Bottom Slope on the Strong Cyclonic Motion. In this section, we would like to investigate the effect of sloping bottom on the strong cyclonic motions. For convenience, we choose a typical case of a moderate tropical cyclone as our prototypical problem and seek for its possible model vortex $B$ that is dynamically similar in the laboratory. For example, let us choose a strong cyclonic vortex with a maximum azimuthal speed $V_{m}=40 \mathrm{~m} / \mathrm{s}$ and its corresponding radius $R_{m}=150 \mathrm{~km}$ initializing at the latitude of $20^{\circ}$ in the Northern Hemisphere. The unperturbed layer depth $H_{0}$ is about $10 \mathrm{~km}$ and a maximum vortex depression $\eta_{v}$ is assumed to be $295.56 \mathrm{~m}$. We assume that the vortex shape parameter $B$ of the prototypical cyclone is 1.5 , and the vortex size parameter $A$ can be determined as $1837 \mathrm{~km}$ by (23). On the laboratory side, we choose a typical strong vortex with maximum azimuthal speed $V_{m}=6 \mathrm{~cm} / \mathrm{s}$ and a corresponding radius $R_{m}=3 \mathrm{~cm}$ moving on a sloping bottom with $s_{y}=0.0538$ in a rotating tank with an angular speed $\Omega=0.785 \mathrm{rad} / \mathrm{s}$. Under the similarity condition (14), the unperturbed depth $H_{0}$ in the tank can be obtained as $10.47 \mathrm{~cm}$. The maximum depression depth $\eta_{v}$ of the vortex in the laboratory can also be determined from the similarity condition (15) as $0.206 \mathrm{~cm}$.

Figure 9 shows the numerical results of trajectory of vortex $B$ translating on a sloping bottom with four different slopes as 0.01076 (Case S1), 0.0538 (Case S2), 0.1345 (Case S3), and 0.269 (Case S4). The trajectories were determined from the calculated streamlines, and the origin of reference was located $60 \mathrm{~cm}$ north from the south bound of the tank and $60 \mathrm{~cm}$ west from the east bound of the tank. Comparing the above four vortex paths under different bottom slopes, we summarize as follows. First, the vortices in all examples generally move to the northwest. All the examples in the first half of the path show linear motion, when the vortex maintains a single vortex structure. The moving speed of the vortex is proportional to the slope of the bottom. The greater the slope is, the faster the vortex moves. Second, the vortex in
Case S1 moves at the slowest speed and continues to move slowly towards the northwest as a single vortex. On the other hand, for Cases S2, S3, and S4, the vortices accelerate significantly at the early stage and decelerate gradually at the following stage. These trends of intensity change and translating speed are basically coincided with the theory proposed by Resnik et al. [10]. That is to say, in the initial stage, the vortex is between the advection time scale and the wave time scale, so it is accelerated by beta-gyres. However, after wave time scale, beta-gyres will further induce secondary beta-gyres due to nonlinearity. This process will last for a long time to a certain time scale and cause the vortex to slow down.

Figure 10 shows the streamline graphs for Case S3. We can observe that several secondary vortices at the east of the primary vortex were induced consequently by strong planetary $\beta$ effect. As a result, these secondary vortices in turn interact with the primary vortex and cause a slight meandering and a distortion of the primary vortex. These waves demonstrate an alternative pattern of clockwise and anticlockwise circulation cells translating westward and they are usually referred to as topographic Rossby wave as mentioned in [33].

Figure 11 demonstrates the vorticity contours for Case S4. Prominent Rossby wave wakes following the primary vortex were excited by steep bottom slope. This fact shows that the induced Rossby wave wakes not only alter the trajectory of the primary vortex (as shown in Figure 9) but also stretch the primary vortex from axis-symmetric to axisasymmetric ( $t=36 \mathrm{~s}$ to $t=56 \mathrm{~s}$ as shown in Figure 10). The understanding of this stage can be explained by the vortex distortion time scale proposed by Resnik et al. [10]. In Figure 11, a negative vorticity patch $\mathrm{N} 1$ was generated in the vicinity of the primary vortex $\mathrm{P} 1$ and was developed to a vorticity tendril during $t=0 \mathrm{~s}$ to $16 \mathrm{~s}$. From $t=16 \mathrm{~s}$ to $32 \mathrm{~s}$, this vorticity tendril was influenced and has been stretched by following positive vorticity patch $\mathrm{P} 2$ which formed 


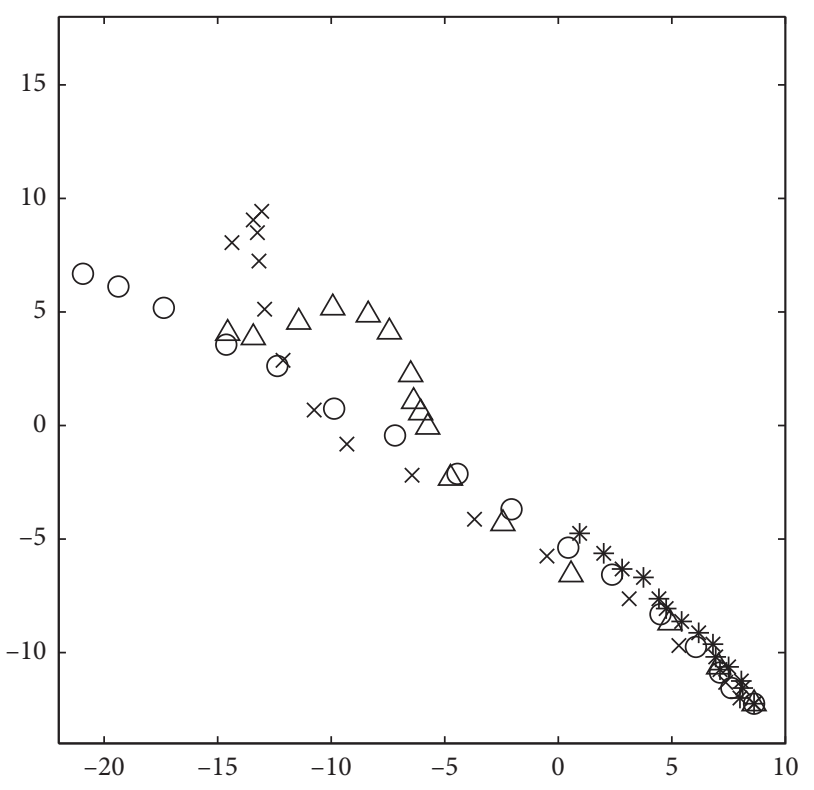

Figure 9: Simulation tracks of a benchmark vortex $B$ translating on a sloping bottom. The vortices were indicated by asterisks, circles, crosses, and triangles for tracks on different bottom slopes of $0.01076,0.0538,0.1345$, and 0.269 , respectively.

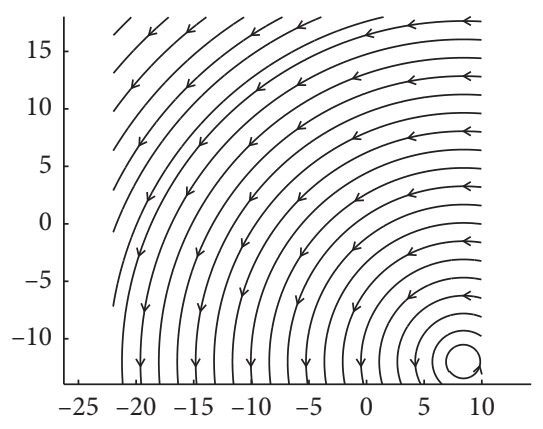

(a)

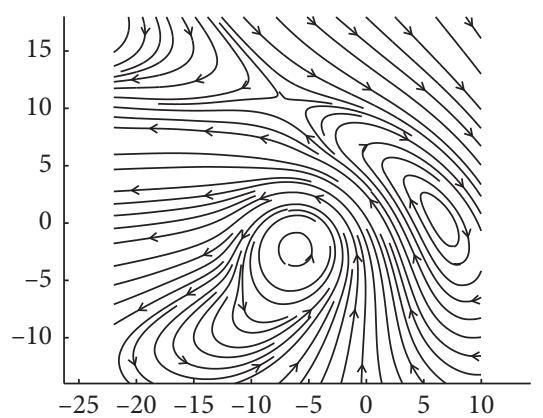

(d)

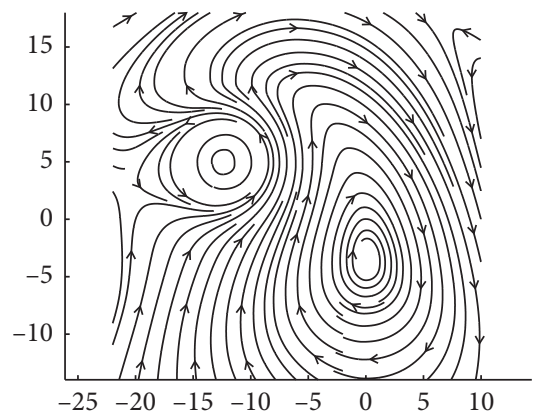

(g)

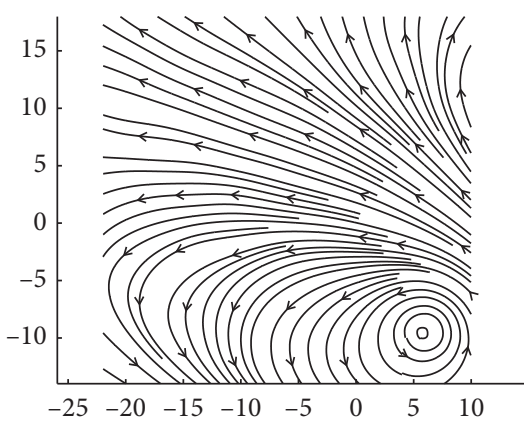

(b)

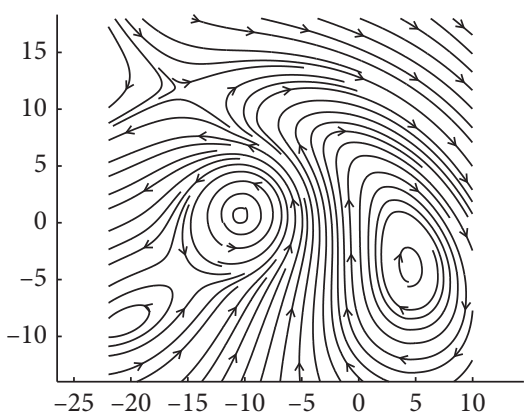

(e)

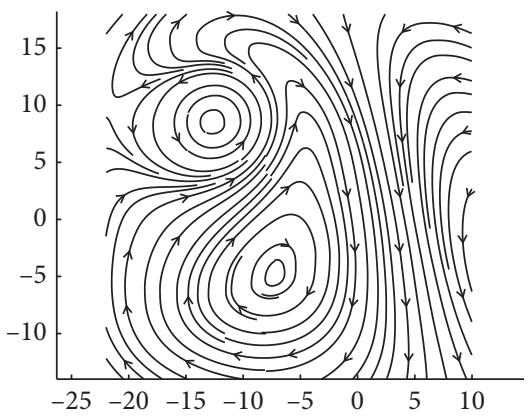

(h)

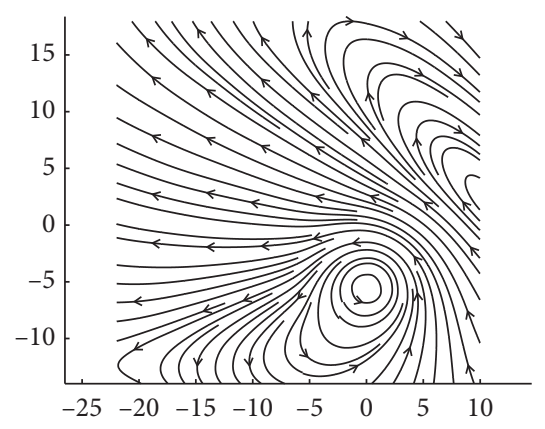

(c)

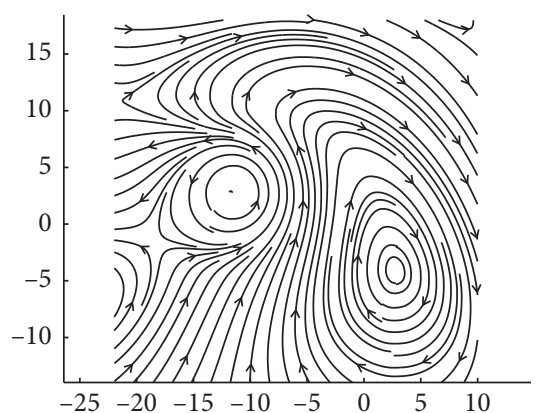

(f)

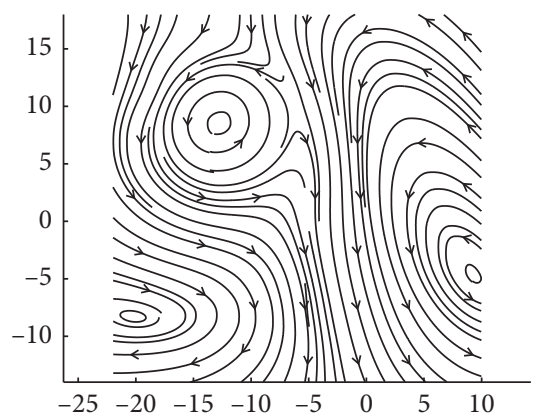

(i)

Figure 10: Streamlines plots of Case S3 with a bottom slope $s_{y}=0.1345$. (a) $t=0 \mathrm{~s}$, (b) $t=8 \mathrm{~s}$, (c) $t=16 \mathrm{~s}$, (d) $t=24 \mathrm{~s}$, (e) $t=32 \mathrm{~s}$, (f) $t=36 \mathrm{~s}$, (g) $t=40 \mathrm{~s}$, (h) $t=48 \mathrm{~s}$, and (i) $t=56 \mathrm{~s}$. 


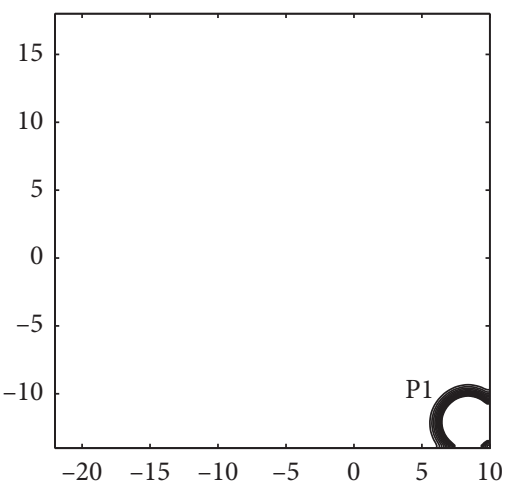

(a)

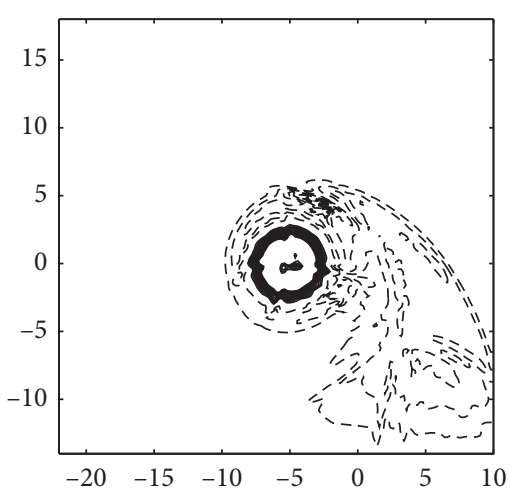

(d)

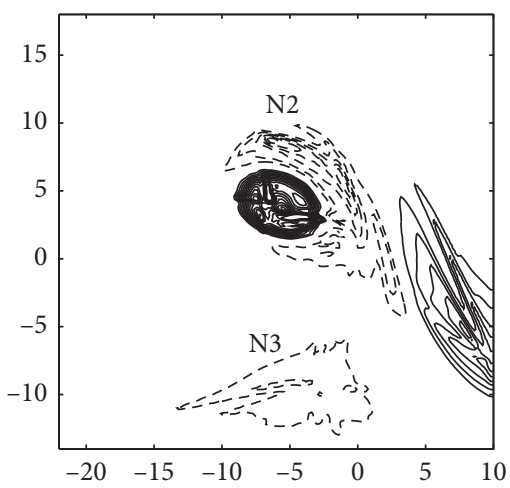

(g)

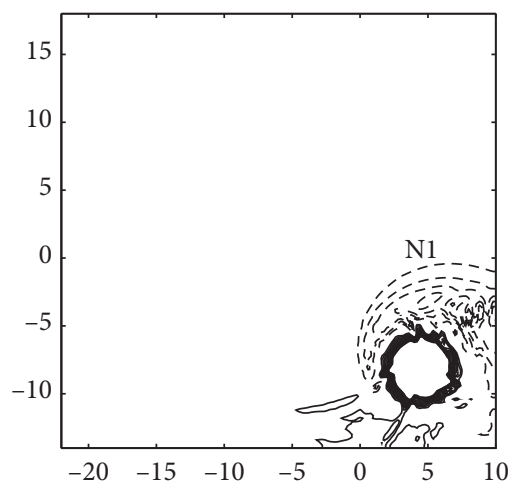

(b)

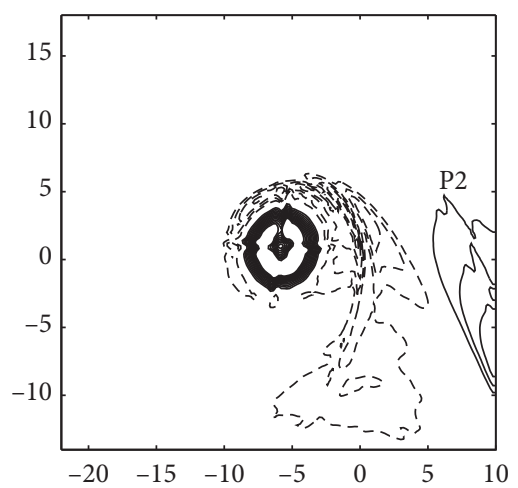

(e)

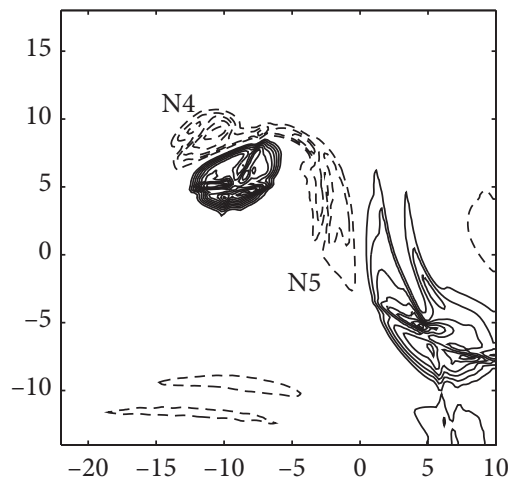

(h)

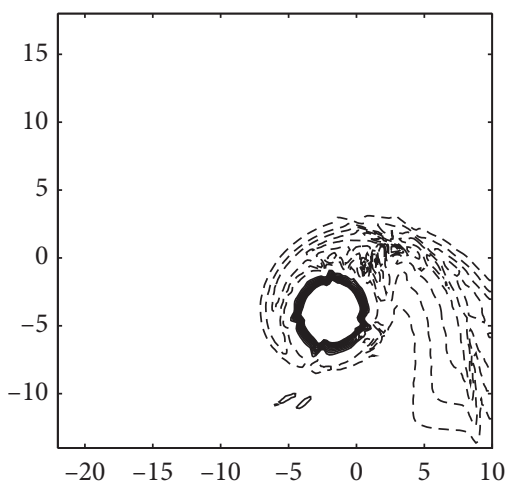

(c)

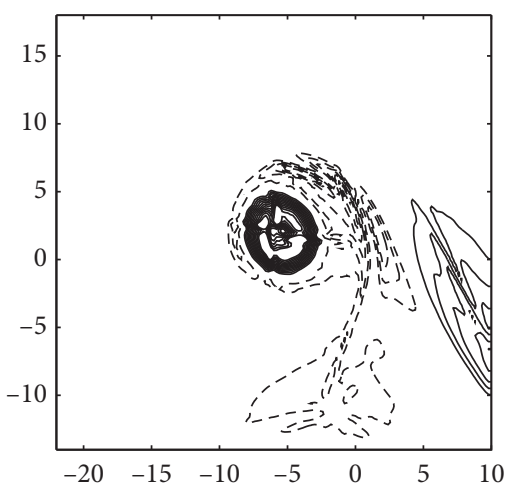

(f)

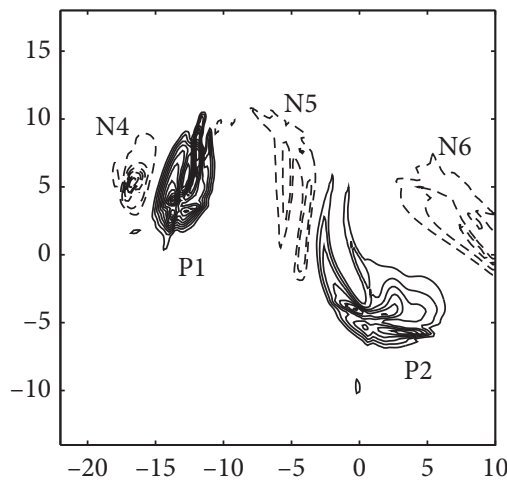

(i)

Figure 11: Contours of relative vorticity of Case S4 with a bottom slope $s_{y}=0.269$. (a) $t=0 \mathrm{~s}$, (b) $t=8 \mathrm{~s}$, (c) $t=16 \mathrm{~s}$, (d) $t=24 \mathrm{~s}$, (e) $t=32 \mathrm{~s}$, (f) $t=36 \mathrm{~s}$, (g) $t=40 \mathrm{~s}$, (h) $t=48 \mathrm{~s}$, and (i) $t=56 \mathrm{~s}$.

behind N1. Generally, it is shown that the simulation by MSWM well demonstrates the entire flow features. It also reveals complicated nonlinear interactions of the vortex and its induced Rossby wave wakes that consist of vorticity patches with alternative signs ( $\mathrm{P}$ for positive and $\mathrm{N}$ for negative, for short).

In addition, from the point of view of total energy conservation, it can be observed in Figure 11 that the Rossby waves radiated from the primary vortex provide the energy required for wake development. This process mainly occurs in the near field of vortex, namely, beta-gyres. The wake coexists and remains near the primary vortex and keeps a quasi-steady translation speed similar to that of the primary vortex [10]. This interpretation is different from the viewpoint held in some literatures $[8,34]$. They explained that the Rossby wave wake is the product of quasiresonance between the primary vortex and planetary vorticity gradient. However, the conservation of the total energy of the system does not support this argument and needs further study.

\section{Conclusions}

In this study, a joint theoretical and numerical study is used to investigate the flow features of a strong cyclonic vortex generated in a rotating tank with a sloping bottom. This study clarifies the idea of the dynamical similarity between the prototypical and model flow fields by satisfying the similarity conditions (14) and (15). Calculations by the 
proposed modified shallow water model for the strong and intense cyclonic motions show a close agreement with the experimental results in a rotating tank. The present study proposed a modified shallow water model incorporating a gradient-wind-balance (GWB) vortex model for investigating the hurricane-like cyclonic motions on a $\beta$-plane in the Northern Hemisphere and their structures in a rotating tank with a gently sloping bottom.

There are two main advantages of the model. (i) Unlike the traditional QGVE model, the MSWM is more suitable to take care of the significant depth depression of the vortex, which is a prominent feature of large Rossby number hurricane-like vortices. From the surface depression measurements, the effect of vortex stretching owing to this vortex depression had the same order of magnitude as that of the vortex stretching caused by the sloping bottom. (ii) Another significant source of vortex stretching that should be considered was the parabolic free surface resulting from the tank rotation. In the present MSWM model, this effect of paraboloidal free surface was conveniently represented by an effective gravity. Regarding the simulation of the large Rossby number vortices, the GWB vortex model pictures the vortex structure more accurately than the traditional Gaussian/Rankine vortex models. It is noted that, in literature, for vortices with both large Rossby number $\left(\mathrm{Ro}_{v} \approx O(1)\right)$ and large Burger number $(\mathrm{Bu} \gg 1)$, the fast motion in the flow field, that is, inertia gravity wave (IGW), may be decoupled and can be emitted from the slow vortex motion [34, 35]. The Burger number is defined as $\mathrm{Bu}=\left(R_{d}^{2} / R_{m}^{2}\right)$, where $R_{d}$ is the Rossby deformation radius as $R_{d}=\sqrt{g H_{0}} / f_{0}$. Take the vortex $S$ as an example, where $\mathrm{Ro}_{v}=4.32$ and $\mathrm{Bu} \approx 1,154$. Although the flow field of vortex $S$ satisfies the conditions of IGW generation, there is no direct evidence of the emission of IGW in the current experiment or numerical results. This interesting phenomenon is worthy of further and careful study in the future.

Our major results obtained in this paper were presented in two parts. In the first part, a numerical simulation of a monopolar vortex translating on a gentle-slope bottom was carried out to verify the experimental results in the previous study [22]. Close agreements were found between experiment and simulation, including the streamline patterns and the vortex trajectory. After the long-term evolution, the coherency and monopolar nature of a strong vortex $\left(\mathrm{Ro}_{v} \approx O(1)\right)$ remained both in the experimental and numerical results. In the second part, the long-term behaviours of vortex motion on different sloping bottoms were investigated numerically. For those gentle-slope cases (Case S1 and Case S2), the vortex moves steadily to the northwest. As the bottom slope goes steeper (Case S3 and Case S4), the trajectory of the primary vortex is being influenced by the associate secondary vortices and altered as meandered, curved motion. In the case of steepest slope (S4), the interaction between the primary vortex and the induced Rossby wave wakes reveals many interesting features such as the deformation of primary vortex, the emergence of a dipolar vortex, and the associated Rossby wave wakes. The interaction between strong cyclonic vortices and the accompanied secondary circulation structures under various flow conditions requires comprehensive exploration through theoretical analysis, numerical simulation, and laboratory experiments. They may provide invaluable ingredients for understanding the physics of intense oceanic eddies [33, 36] or atmospheric vortices [37] with coherent wave trains [38] and they will be reported elsewhere.

\section{Data Availability}

No data were used to support this study.

\section{Conflicts of Interest}

The authors declare that they have no conflicts of interest.

\section{Acknowledgments}

The authors acknowledge Shandong Polytechnic, China, for the partial support of this research. The first author thanks Professor C. C. Chu and Professor C. C. Chang of National Taiwan University for their supports in the early stage of this work.

\section{References}

[1] J. Adem, "A series solution for the barotropic vorticity equation and its application in the study of atmospheric vortices," Tellus, vol. 8, no. 3, pp. 364-372, 1956.

[2] G. R. Flierl, "The application of linear quasigeostrophic dynamics to Gulf Stream rings," Journal of Physical Oceanography, vol. 7, no. 3, pp. 365-379, 1977.

[3] J. C. McWilliams and G. R. Flierl, "On the evolution of isolated, nonlinear vortices," Journal of Physical Oceanography, vol. 9, pp. 1155-1182, 1979.

[4] M. Fiorino and R. L. Elsberry, "Some aspects of vortex structure related to tropical cyclone motion," Journal of the Atmospheric Sciences, vol. 46, pp. 975-990, 1989.

[5] G. M. Reznik, "Dyanmics of singular vortices on a betaplane," Journal of Fluid Mechanics, vol. 240, pp. 405-432, 1992.

[6] G. M. Reznik and W. K. Dewar, "An analytical theory of distributed axisymmetric barotropic vortices on the $\beta$-plane," Journal of Fluid Mechanics, vol. 269, pp. 301-321, 1994.

[7] G. G. Sutyrin and G. R. Flierl, "Intense vortex motion on the beta plane: development of the beta gyres," Journal of the Atmospheric Sciences, vol. 51, no. 5, pp. 773-790, 1994.

[8] G. K. Korotaev and A. B. Fedotov, "Dynamics of an isolated barotropic eddy on a beta-plane," Journal of Fluid Mechanics, vol. 264, pp. 277-301, 1994.

[9] S. G. Llewellyn Smith, "The motion of a non-isolated vortex on the beta-plane," Journal of Fluid Mechanics, vol. 346, pp. 149-179, 1997.

[10] G. M. Reznik, R. Grimshaw, and E. S. Benilov, "On the longterm evolution of an intense localized divergent vortex on the beta-plane," Journal of Fluid Mechanics, vol. 422, pp. 249-280, 2000.

[11] E. Firing and R. C. Beardsley, "The behavior of a barotropic eddy on a $\beta$-plane," Journal of Physical Oceanography, vol. 6, no. 1, pp. 57-65, 1976.

[12] M. Takematsu and T. Kita, "The behavior of isolated free eddies in a rotating fluid: laboratory experiment," Fluid Dynamics Research, vol. 3, no. 1-4, pp. 400-406, 1988. 
[13] A. Masuda, K. Marubayashi, and M. Ishibashi, "A laboratory experiment and numerical simulation of an isolated barotropic eddy in a basin with topographic $\beta$," Journal of Fluid Mechanics, vol. 213, no. 1, pp. 641-655, 1990.

[14] G. F. Carnevale, R. C. Kloosterziel, and G. J. F. van Heijst, "Propagation of barotropic vortices over topography in a rotating tank," Journal of Fluid Mechanics, vol. 233, pp. 119-139, 1991.

[15] J.-B. Flór and I. Eames, "Dynamics of monopolar vortices on a topographic beta-plane," Journal of Fluid Mechanics, vol. 456, pp. 353-376, 2002.

[16] E. J. Hopfinger and G. J. F. V. Heijst, "Vortices in rotating fluids," Annual Review of Fluid Mechanics, vol. 25, no. 1, pp. 241-289, 1993.

[17] G. J. F. V. Heijst and H. J. H. Clercx, "Laboratory modelling of geophysical vortices," Annual Review of Fluid Mechanics, vol. 41, pp. 143-164, 2008.

[18] J. S. Lam and D. G. Dritshel, "On the beta-drift of an initial circular vortex patch," Journal of Fluid Mechanics, vol. 436, pp. 107-129, 2001.

[19] S. Kravtsov and G. Reznik, "Numerical solutions of the singular vortex problem," Physics of Fluids, vol. 31, Article ID 066602, 2019.

[20] G. M. Reznik and S. V. Kravtsov, "Singular vortices on a betaplane: a brief review and recent results," Physical Oceanography, vol. 27, pp. 659-676, 2020.

[21] M. M. Jalali and D. G. Dritschel, "Stability and evolution of two opposite-signed quasi-geostrophic shallow-water vortex patches," Geophysical \& Astrophysical Fluid Dynamics, pp. 1-27, 2020.

[22] H. C. Chen, J. H. Leu, Y. L. Lin et al., "Cyclonic motion and structure in rotating tank: experiment and theoretical analysis," Sensors and Materials, 2021.

[23] C. Schär and R. B. Smith, "Shallow-water flow past isolated topography. Part I: vorticity production and wake formation," Journal of the Atmospheric Sciences, vol. 50, no. 10, pp. 1373-1400, 1993.

[24] C. Schär and R. B. Smith, "Shallow-water flow past isolated topography. Part II: transition to vortex shedding," Journal of the Atmospheric Sciences, vol. 50, no. 10, pp. 1401-1412, 1993.

[25] P. K. Smolarkiewicz, "A fully multidimensional positive definite advection transport algorithm with small implicit diffusion," Journal of Computational Physics, vol. 54, no. 2, pp. 325-362, 1984.

[26] P. K. Smolarkiewicz and T. L. Clark, "The multidimensional positive definite advection transport algorithm: further development and applications," Journal of Computational Physics, vol. 67, no. 2, pp. 396-438, 1986.

[27] P. K. Smolarkiewicz and L. G. Margolin, "MPDATA: a finitedifference solver for geophysical flows," Journal of Computational Physics, vol. 140, no. 2, pp. 459-480, 1998.

[28] P. K. Smolarkiewicz, "Multidimensional positive definite advection transport algorithm: an overview," International Journal for Numerical Methods in Fluids, vol. 50, no. 10, pp. 1123-1144, 2006.

[29] J. Pedlosky, Geophysical Fluid Dynamics, Springer, Berlin, Germany, 2nd edition, 1986.

[30] M. V. Nezlin and E. N. Snezhkin, Rossby Vortices, Spiral Structures, Solitons: Astrophysics and Plasma Physics in Shallow Water Experiments, Springer-Verlag, Berlin, Germany, 1993.

[31] H. C. Davies, "Limitations of some common lateral boundary schemes used in regional NWP models," Monthly Weather Review, vol. 111, no. 5, pp. 1002-1012, 1983.
[32] G. J. Holland, "An analytic model of the wind and pressure profiles in hurricanes," Monthly Weather Review, vol. 108, no. 8, pp. 1212-1218, 1980.

[33] L. Zavala Sansón and G. J. F. V. Heijst, "Laboratory experiments on flows over bottom topography," Modeling Atomospheric and Oceanic Flows: Insights from Laboratory Experiments and Numerical Simulations, pp. 139-158, 2015.

[34] V. Zeitlin, "Decoupling of balanced and unbalanced motions and inertia-gravity wave emission: small versus large Rossby numbers," Journal of the Atmospheric Sciences, vol. 65, no. 11, pp. 3528-3542, 2008.

[35] V. Zeitlin, Geophysical Fluid Dynamics: Understanding (Almost) Everything with Rotating Shallow Water Models, Oxford University Press, Oxford, UK, 2018.

[36] G. R. Flierl, "Rossby wave radiation from a strongly nonlinear warm eddy," Journal of Physical Oceanography, vol. 14, no. 1, pp. 47-58, 1984.

[37] J. M. Cosgrove and L. K. Forbes, "Nonlinear behaviour of interacting mid-latitude atmospheric vortices," Journal of Engineering Mathematics, vol. 104, no. 1, pp. 41-62, 2017.

[38] K. D. Krouse, A. H. Sobel, and L. M. Polvani, "On the wavelength of the Rossby waves radiated by tropical cyclones," Journal of the Atmospheric Sciences, vol. 65, no. 2, pp. 644-654, 2008. 